\title{
A fatal case of a ruptured cerebral aneurysm detected by postmortem computed tomography angiography using a new contrast-medium solution
}

Stamou, Stamatios ; Gascho, Dominic ; Eggert, Sebastian ; Berger, Florian ; Thali, Michael J ; Flach, Patricia M

\begin{abstract}
Forensic autopsy is still the criterion standard in legal investigations. Currently, notable transformation is occurring because postmortem imaging is being increasingly endorsed. This article highlights the effectiveness of postmortem computed tomography angiography by using a new contrast medium mixture as a solitary tool to define the cause and manner of death in a ruptured cerebral aneurysm case. Based on the imaging results, autopsy was not mandated and the case was closed.
\end{abstract}

DOI: https://doi.org/10.1097/PAF.0000000000000259

Posted at the Zurich Open Repository and Archive, University of Zurich

ZORA URL: https://doi.org/10.5167/uzh-126261

Journal Article

Published Version

Originally published at:

Stamou, Stamatios; Gascho, Dominic; Eggert, Sebastian; Berger, Florian; Thali, Michael J; Flach, Patricia M (2016). A fatal case of a ruptured cerebral aneurysm detected by postmortem computed tomography angiography using a new contrast-medium solution. American Journal of Forensic Medicine and Pathology, 37(4):227-230.

DOI: https://doi.org/10.1097/PAF.0000000000000259 


\title{
A Fatal Case of a Ruptured Cerebral Aneurysm Detected by Postmortem Computed Tomography Angiography Using a New Contrast-Medium Solution
}

\author{
Stamatios Stamou, MD, Dominic Gascho, Sebastian Eggert, MD, Florian Berger, MD, \\ Michael J. Thali, MD, MBA, and Patricia M. Flach, MD
}

\begin{abstract}
Forensic autopsy is still the criterion standard in legal investigations. Currently, notable transformation is occurring because postmortem imaging is being increasingly endorsed. This article highlights the effectiveness of postmortem computed tomography angiography by using a new contrast medium mixture as a solitary tool to define the cause and manner of death in a ruptured cerebral aneurysm case. Based on the imaging results, autopsy was not mandated and the case was closed.
\end{abstract}

Key Words: postmortem CT, virtopsy, postmortem CT angiography, $\mathrm{SAH}$, aneurysm, new contrast medium solution for postmortem CT angiography

(Am J Forensic Med Pathol 2016;00: 00-00)

S ubarachnoid hemorrhage ( $\mathrm{SAH}$ ) is a known cause of mortality $S$ in younger populations. ${ }^{1}$ There is a well-established connection between this pathological entity with ruptured brain aneurysms, which are responsible for approximately $80 \%$ to $85 \%$ of cases. ${ }^{2}$

In common clinical practice, the investigation of nontraumatic SAH requires radiological imaging of the brain with visualization of the vasculature to detect the underlying pathology. Computed tomography (CT) is primarily used due to the ease of accessibility in emergency departments and the relatively rapid establishment of the diagnosis compared with other imaging modalities such as magnetic resonance imaging and digital subtraction angiography. ${ }^{3}$

The major cause of mortality in untreated SAH cases is central regulatory failure, which is primarily due to a mass effect by cerebral edema. In forensic pathology, it is important to differentiate this process from other causes that can lead to the same result, such as ischemia, tumors, or secondary failure, that is, cerebellar bleeding.

Over the last decade, many forensic institutes have implemented postmortem CT (PMCT) into the daily routine either as a triage, supplement, or even replacement for autopsy. ${ }^{4}$ Multiple approaches for PMCT angiography (PMCTA) are currently used to investigate the vasculature based on the minimally invasive PMCT. ${ }^{5,6}$ The literature describes different contrast medium mixtures for PMCTA. All of the mixtures contain iodine contrast medium that is usually the same as the medium used in clinical patients, but differs in the carrier substance. Initially diesel oil was

Manuscript received March 29, 2016; accepted May 15, 2016.

From the Institute of Forensic Medicine, University of Zurich, Zurich, Switzerland.

The authors report no conflict of interest.

Reprints: Stamatios Stamou, MD, Institute of Forensic Medicine, Virtopsy, University of Zurich, Winterthurerstrasse 190/52, 8057 Zurich, Switzerland.

E-mail: stamatios.stamou@irm.uzh.ch.

Copyright (C) 2016 Wolters Kluwer Health, Inc. All rights reserved.

ISSN: 0195-7910/16/0000-0000

DOI: 10.1097/PAF.0000000000000259 used. ${ }^{7}$ Currently, components are described as polyethylene glycol (PEG), paraffin oil, or simply sodium chloride $(\mathrm{NaCl})$, each of which has several advantages and drawbacks. ${ }^{6,8}$ Therefore, research concerning the best usable contrast medium mixture for forensic PMCTA is ongoing.

Although forensic radiological imaging is increasingly emerging, autopsy is still considered the criterion standard for forensic investigations. Hence, research, education, and establishing guidelines for forensic radiology with its worldwide incremental usage is of paramount importance..$^{9-12}$ Postmortem CT is acknowledged for the detection of fractures, gas embolism, and foreign material, and PMCTA is used to address certain questions regarding vasculature and the origins of the hemorrhage. ${ }^{13-16}$ Therefore, PMCTA plays an important role in forensic imaging of the vasculature.

This article highlights the effectiveness of PMCTA using a new contrast medium mixture as a solitary tool to define the cause and manner of death in a ruptured cerebral aneurysm case.

\section{Case History}

A 50-year-old woman with no known medical history was found dead by the police in the late afternoon hours in her apartment. The door was locked, and the authorities entered the housing through the balcony. The naked woman was lying in a supine position on the floor next to the bathroom entrance. At the scene, external inspection did not show any traumatic injuries or any sign of external influence. The body was transferred to the forensic institute for clarification of the cause and manner of death and underwent routine PMCT imaging before the initial autopsy mandated by the state attorney.

\section{MATERIALS AND METHODS}

\section{Contrast Medium Mixture for PMCTA}

The responsible justice department approved this examination. The contrast agent mixture used in this study consisted of an infusion solution of Ringer's acetate similar in compounds to Jonosteril (Fresenius, Oberdorf, Switzerland) and water-soluble ioversol (Optiray 300; Guerbet, Paris, France) at a 17:1 ratio (resulting in a radiodensity of 300-350 HU; $60 \mathrm{~mL}$ of iodine contrast agent in $1000 \mathrm{~mL}$ of carrier substance). The flow rate had a mean of $0.6 \mathrm{~L} / \mathrm{min}$ and a maximum of $2.5 \mathrm{~L} / \mathrm{min}$, and the volumes were $2000 \mathrm{~mL}$ (arterial injection) and $1500 \mathrm{~mL}$ (venous injection). The precise protocol and preparation of the PMCTA was performed as described in the literature. ${ }^{17}$

\section{PMCT and PMCTA Imaging}

For postmortem imaging, the body was wrapped in an impermeable body bag to prevent contamination of the equipment, especially during PMCTA. Unenhanced PMCT and PMCTA were performed using a 128-slice CT scanner (SOMATOM Flash 


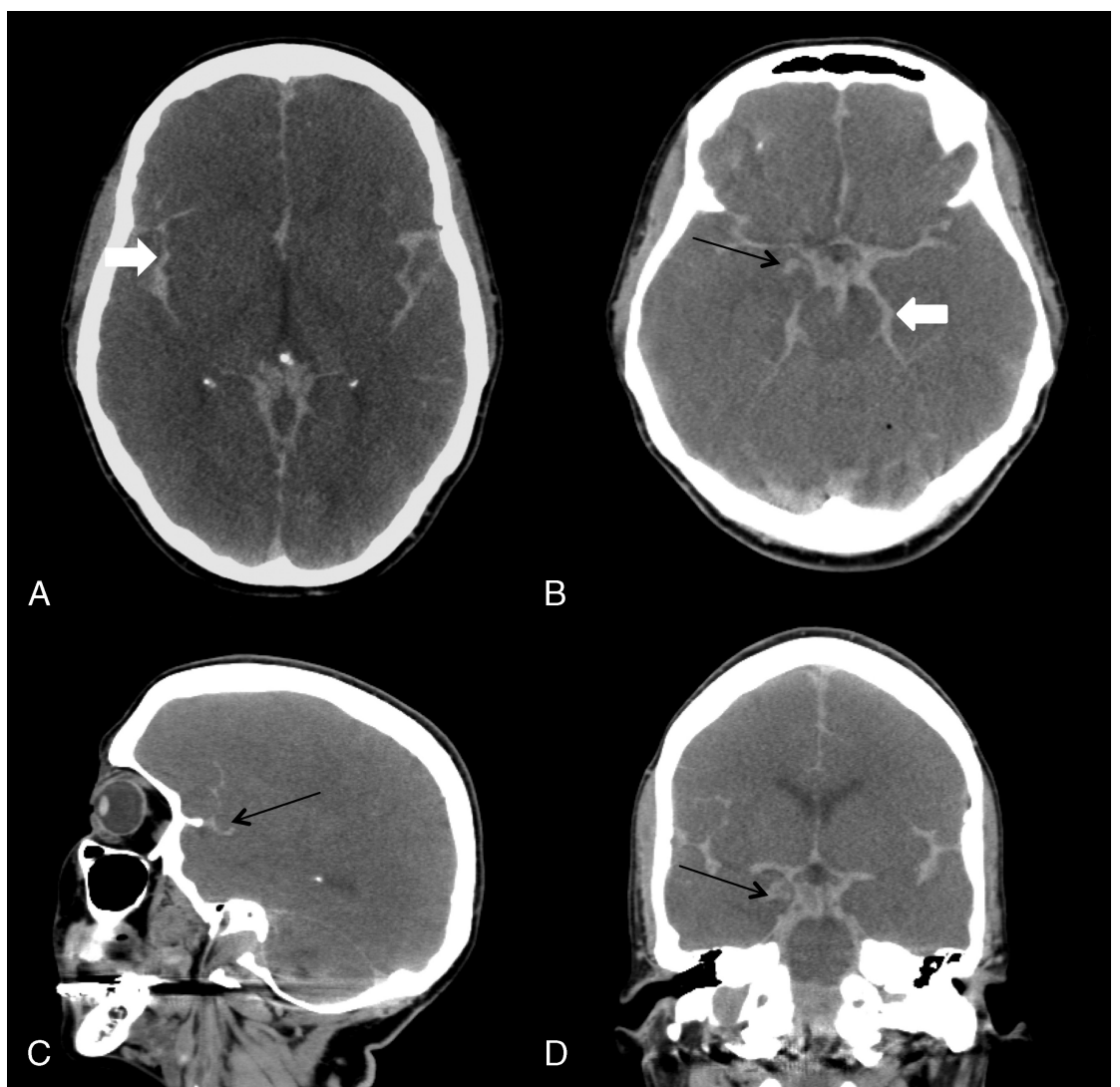

FIGURE 1. Unenhanced PMCT in an axial (A and B), sagittal (C), and coronal (D) plane depicting extensive SAH. Note the focally increased hemorrhage accumulation (marked by a black arrow, B-D) adjacent to the circle of Willis on the right. A, Subarachnoid bleeding in the sylvian fissure (white thick arrow) and along the cerebral falx. B, Subarachnoid bleeding in the interpeduncular cistern and the ambient cistern (exemplarily white arrow). C, Effacement of the sulci and loss of corticomedullary differentiation by brain edema due to typical postmortem changes and cerebral edema. D, Effacement of the temporal horns of the side ventricles by brain edema.

Definition; Siemens Medical Solutions, Forchheim, Germany). The general scan parameters were applied as recommended by the literature ${ }^{17}: 120 \mathrm{kVp}$ (PMCT) and $100 \mathrm{kVp}$ (PMCTA) tube voltage, 350 effective $\mathrm{mA}$ tube current-time product, $1.0 \mathrm{~mm}$ slice thickness, $0.5 \mathrm{~mm}$ increment, and reconstructions in soft and hard kernels, with a medium-hard kernel for PMCTA with the according windows. The head and neck scan was calculated from the scan with lowered arms in an adjusted field of view (maximum, $300 \mathrm{~mm}$ ) with a medium soft kernel in an angio window with a slice thickness of $1 \mathrm{~mm}$ and an increment of $0.5 \mathrm{~mm}$. The entire PMCTA took, from transportation to scan and back to the cooling storage, about 30 minutes.

\section{Image Analysis}

The primary image review and multiplanar reconstructions were performed using a CT workstation (Leonardo; Siemens, Medical Solutions, Forchheim, Germany). A multimodality workstation was used for the radiological assessment (Syngo.via, Version VA30A; Siemens, Medical Solutions, Erlangen, Germany). Image reconstructions were performed with a cinematic rendering software with a physics-based rendering technique (Research Platform, Cinematic Rendering, Siemens CT 1.0.0, Frontier Prototype Store, Syngo.via, Version VA30A; Siemens, Medical Solutions, Erlangen, Germany). Radiological analysis and reporting were performed by 2 experienced board-certified forensic radiologists, one of whom was board-certified for neuroradiology.

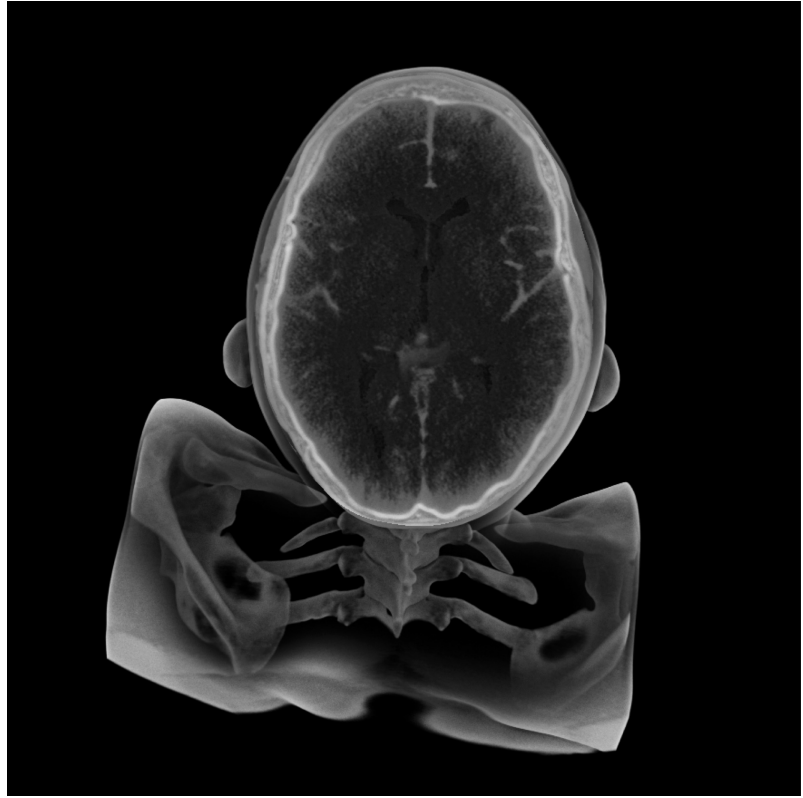

FIGURE 2. This image visualizes the extent of the SAH on an unenhanced PMCT. The 3D reconstruction (Research Platform, Cinematic Rendering, Siemens CT 1.0.0, Frontier Prototype Store) with the view from posterior and above into the virtually clipped skull with visualization of the brain parenchyma. 


\section{RESULTS}

\section{Imaging}

The PMCT showed extensive subarachnoid bleeding in the supratentorial sulci and the basal cisterns with signs of an accompanying generalized antemortem agonal edema concomitant with the expected typical postmortem swelling as shown in Figure 1 and Figure 2. The PMCTA revealed a broad-based ruptured aneurysm of the supraclinoid segment of the right internal carotid artery (Figs. 3, 4). The size of the aneurysm could not be accurately defined due to the extensive contrast extravasation from the ruptured aneurysm, which was also detectable on PMCTA (Fig. 3). No additional abnormalities of the vessels or forensically relevant pathologies of the deceased were detected. Imaging revealed a natural cause of death due to central regulatory failure in a masseffective SAH in the course of a ruptured cerebral aneurysm.

\section{Contrast Medium Mixture}

The contrast agent mixture used in this study was easier to administer because the viscosity of the carrier substance was lower than the typically used PEG. ${ }^{6}$ The image contrast was

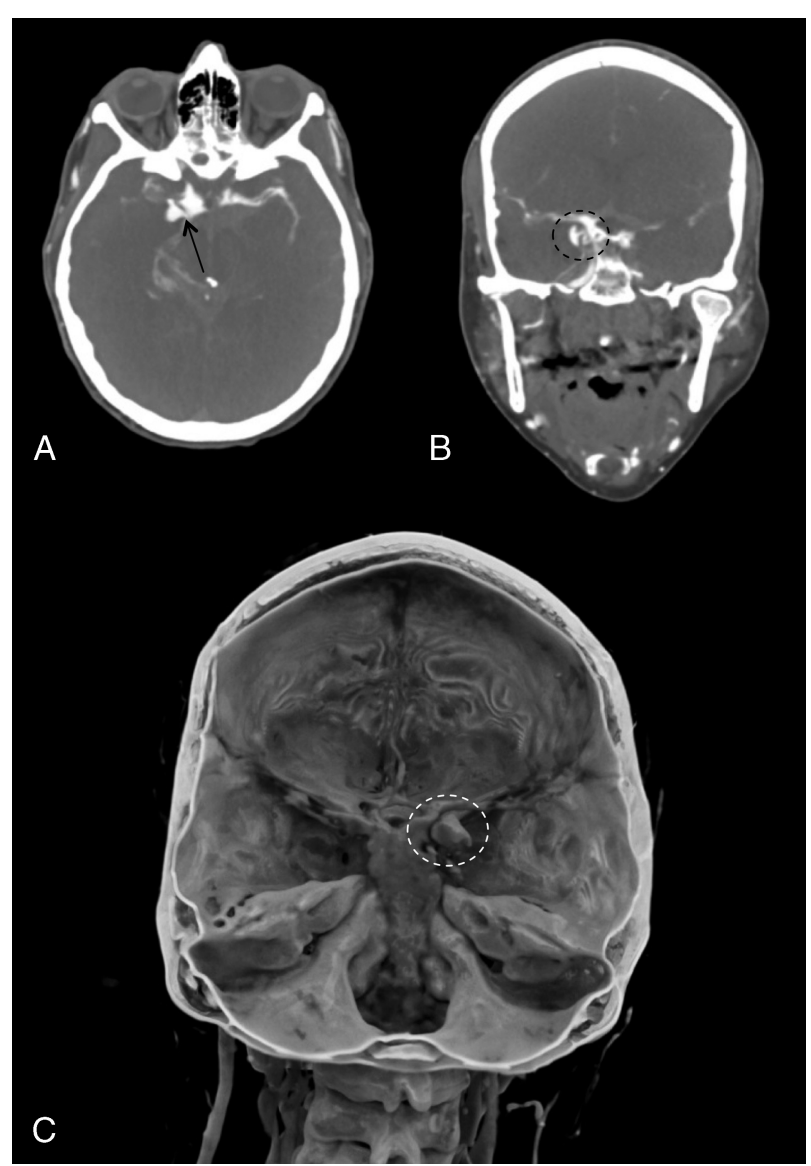

FIGURE 3. Axial (A) and coronal (B) maximum intensity projections of the ruptured aneurysm with contrast medium extravasation on the PMCTA. The contrast medium extravasation due to the ruptured aneurysm is marked by a black arrow (A) and a black circle (B). C, The aneurysm is displayed as a 3D reconstruction (Research platform, Cinematic Rendering, Siemens CT 1.0.0, Frontier Prototype Store) with its contrast extravasation (white circle) viewed from posterior into the virtually opened skull.

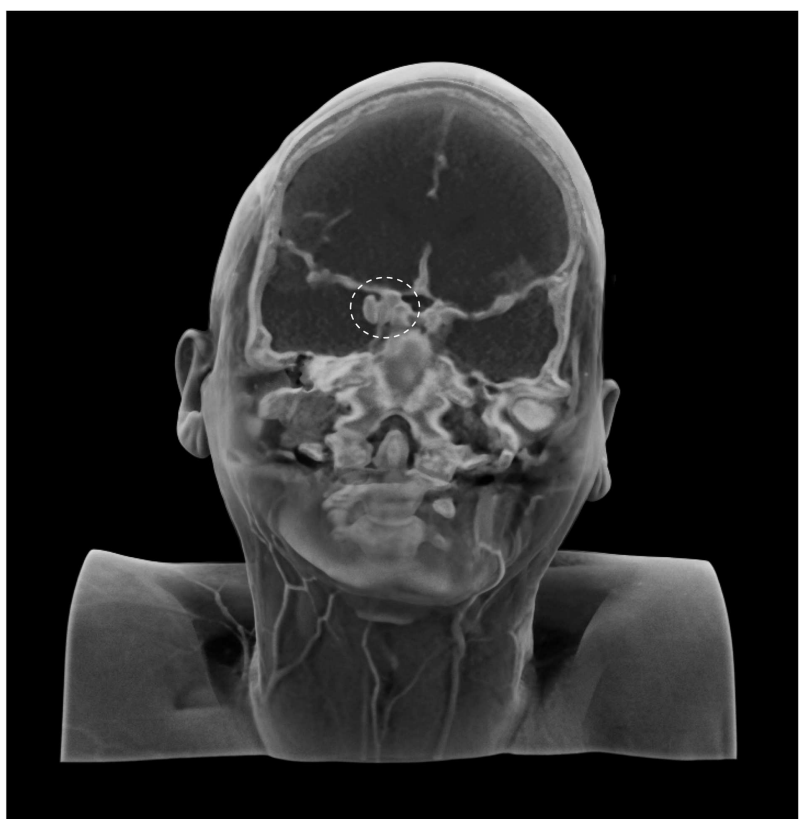

FIGURE 4. Anterior reconstructed display of the ruptured aneurysm (white circle) with its surrounding tissue by Research platform, Cinematic Rendering, Siemens CT 1.0.0, Frontier Prototype Store.

equivalent to the standard contrast medium mixture in measurable Hounsfield Units and in visual assessment. No drawbacks regarding the head and neck region were detected using the new contrast medium mixture.

\section{DISCUSSION}

The case report presents a new technical approach using a new contrast medium mixture for PMCTA and forensic clarification based solely on the radiological findings, thereby highlighting the role of PMCTA in the field of forensic medicine.

PEG-based and paraffin oil-based contrast medium mixtures are currently used in routine examinations. ${ }^{6,7,18,19}$ These contrast medium mixtures have many advantages but also disadvantages such as physical alterations that affect the consistency of parenchymal organs and result in color changes within organ tissues (eg, myocardium with hindered macroscopic detection of myocardial infarction), hardening of the cruor (probably due to osmolarity), and slippery, and therefore impeded handling during dissection. These factors influence the autopsy, the judgment by the forensic pathologists who have to adapt to these "artifacts" created by the applied contrast media mixture during PMCTA. This feedback by autopsy technicians, forensic pathologists, and the involved radiologist's participation during autopsy resulted in the ongoing study to test other carrier substances for PMCTA with fewer alterations of the organs that still provided diagnostic PMCTA images. The use of the new contrast medium mixture resulted visually in an excellent contrast quality of the vessels in the region of interest and a facilitated application due to the lower viscosity and non-oily haptic of the carrier substance. This result indicates that Ringer's acetate could be an addition to the inventory used for PMCTA. However, extravasation may occur in early autolytic tissues such as the mucosa and pancreas, and the extent of these limitations has still to be evaluated. Artifacts in the thorax and abdomen related to the applied PMCTA contrast medium mixture were not considered relevant for this case report because they were not of forensic relevance or contributor to the cause of 
death. This case was one of the first included in an ongoing study investigating new approaches for PMCTA carrier substance. To date, the study is composed of the use of this specific carrier substance collective in up to 10 cases (one of them without autopsy) that mandated after presenting the imaging results. The sole case without autopsy represents this case report.

There is a well-established correlation between SAH and ruptured aneurysms of the circle of Willis and its branches in the literature. Indeed, this phenomenon is considered the most frequent cause of nontraumatic SAH. ${ }^{1,2}$ In the presented case, the death was regarded as a central regulatory failure due to the findings of extensive intracranial bleeding (SAH) and the accompanying antemortem or agonal brain edema. The proof of the ruptured aneurysm with a natural manner of death, in combination with the lack of other suspicious forensic evidence based on the external inspection and further pathological radiological findings made the autopsy redundant. This is one of a recently increasing number of cases where radiological data provided sufficient information regarding the cause and manner of death and thus might serve as triage to distinguish a natural from an unnatural death. ${ }^{20-24}$ Therefore, PMCTA will certainly become requisite in the future and will play an important role in understanding and defining the cause of death.

\section{ACKNOWLEDGMENT}

The authors express their gratitude to Emma Marie Luise Kessler, MD for her generous donation to the Zurich Institute of Forensic Medicine, University of Zurich, Switzerland.

\section{REFERENCES}

1. Johnston SC, Selvin S, Gress DR. The burden, trends, and demographics of mortality from subarachnoid hemorrhage. Neurology. 1998;50:1413-1418.

2. van Gijn J, Rinkel GJ. Subarachnoid haemorrhage: diagnosis, causes and management. Brain. 2001;124:249-278.

3. DeLaPaz RL, Wippold FJ 2nd, Cornelius RS, et al. ACR Appropriateness Criteria ${ }^{\circledR}$ on cerebrovascular disease. J Am Coll Radiol. 2011;8:532-538.

4. Rutty GN, Morgan B, O'Donnell C, et al. Forensic institutes across the world place CT or MRI scanners or both into their mortuaries. J Trauma. 2008;65:493-494

5. Grabherr S, Doenz F, Steger B, et al. Multi-phase post-mortem CT angiography: development of a standardized protocol. Int J Legal Med. 2011;125:791-802.

6. Ross S, Spendlove D, Bolliger S, et al. Postmortem whole-body CT angiography: evaluation of two contrast media solutions. AJR Am J Roentgenol. 2008;190:1380-1389.

7. Grabherr S, Djonov V, Friess A, et al. Postmortem angiography after vascular perfusion with diesel oil and a lipophilic contrast agent. AJR Am J Roentgenol. 2006;187:W515-W523.
8. Grabherr S, Djonov V, Yen $\mathrm{K}$, et al. Postmortem angiography: review of former and current methods. AJR Am J Roentgenol. 2007;188:832-838.

9. The International Society for Forensic Radiology and Imaging (ISFRI). Available at: http://www.isfri.org/. Accessed February 9, 2016.

10. Baglivo M, Winklhofer S, Hatch GM, et al. The rise of forensic and post-mortem radiology - analysis of the literature between the year 2000 and 2011. J Forensic Radiol Imaging. 2013;1:3-9.

11. Flach PM, Thali MJ, Germerott T. Times have changed! Forensic radiology - a new challenge for radiology and forensic pathology. AJR Am J Roentgenol. 2014;202:W325-W334.

12. O'Donnell C, Rotman A, Collett S, et al. Current status of routine post-mortem CT in Melbourne, Australia. Forensic Sci Med Pathol. 2007;3: 226-232.

13. Thali MJ, Braun M, Buck U, et al. VIRTOPSY - scientific documentation, reconstruction and animation in forensic: individual and real $3 \mathrm{D}$ data based geo-metric approach including optical body/object surface and radiological CT/MRI scanning. J Forensic Sci. 2005;50:428-442.

14. Oesterhelweg L, Bolliger SA, Thali MJ, et al. Virtopsy: postmortem imaging of laryngeal foreign bodies. Arch Pathol Lab Med. 2009;133. 806-810.

15. Palmiere C, Binaghi S, Doenz F, et al. Detection of hemorrhage source: the diagnostic value of post-mortem CT-angiography. Forensic Sci Int. 2012;222:33-39.

16. Schulze C, Hoppe H, Schweitzer W, et al. Rib fractures at postmortem computed tomography (PMCT) validated against the autopsy. Forensic Sci Int. 2013;233:90-98

17. Flach PM, Gascho D, Schweitzer W, et al. Imaging in forensic radiology: an illustrated guide for postmortem computed tomography technique and protocols. Forensic Sci Med Pathol. 2014;10:583-606.

18. Grabherr S, Hess A, Karolczak M, et al. Angiofil-mediated visualization of the vascular system by microcomputed tomography: a feasibility study. Microsc Res Tech. 2008;71:551-556.

19. Chevallier C, Doenz F, Vaucher P, et al. Postmortem computed tomography angiography vs. conventional autopsy: advantages and inconveniences of each method. Int J Legal Med. 2013;127:981-989.

20. Flach PM, Ross SG, Bolliger SA, et al. Massive systemic fat embolism detected by postmortem imaging and biopsy. J Forensic Sci. 2012;57: 1376-1380.

21. Franckenberg S, Schulze C, Bolliger SA, et al. Postmortem angiography in computed tomography and magnetic resonance imaging in a case of fatal hemorrhage due to an arterio-venous malformation in the brain. Leg Med (Tokyo). 2015;17:180-183.

22. Addison S, Arthurs OJ, Thayyil S. Post-mortem MRI as an alternative to non-forensic autopsy in foetuses and children: from research into clinical practice. Br J Radiol. 2014;87:20130621.

23. O'Donnell C, Woodford N. Post-mortem radiology — a new sub-speciality? Clin Radiol. 2008;63:1189-1194.

24. Berger N, Ross SG, Ampanozi G, et al. Puzzling over intracranial gas: disclosing a pitfall on postmortem computed tomography in a case of fatal blunt trauma. J Forensic Radiol Imaging. 2013;1:137-141. 